\title{
Endoscopic Submucosal Dissection with/without Adjuvant Therapy for the Treatment of cT1NOMO Esophageal Neoplasms Turned Out to be pT1b Cancers after the Endoscopic Resection
}

Cheng-Han Lee ${ }^{1}$, Mu-Shien Lee ${ }^{1}$,Wen-Yu Chuang ${ }^{2,3}$, Cheng-Hui Lin ${ }^{1,3}$ and Yung-Kuan Tsou ${ }^{* 1,3}$

${ }^{1}$ Department of Gastroenterology and Hepatology, Chang Gung Memorial Hospital, Guishan, Taiwan

${ }^{2}$ Department of Pathology, Chang Gung Memorial Hospital, Guishan, Taiwan

${ }^{3}$ College of Medicine, Chang Gung University, Taoyuan, Taiwan

\section{Abstract}

Background and Aim: After endoscopic submucosal dissection (ESD) for esophageal neoplasms, some patients would have the diagnosis of submucosal (pT1b) cancers. How to manage these patients appropriately after the ESD has not been well elucidated. The aim of this study is to report the outcomes of the patients with pT1b esophageal cancers, with or without adjuvant therapy after the ESD.

Patients and Methods: This is a retrospective observational study. Between May 2013 and November 2017, 23 patients (25 lesions) undergoing ESD with the results of pT1b esophageal squamous cell carcinomas were enrolled in this study. After the ESD, our protocol was to give the patients adjuvant therapy (surgery or chemoradiotherapy) for the following criteria: positive vertical resection margins,lymphovascular (LV) invasion in the resected specimens, and SM2 cancers (tumor invasion to submucosa deeper than $200 \mu \mathrm{m}$ ). Those patients did not meet the criteria or refused adjuvant therapy were followed-up closely.

Results: The mean patient age was 56.3 year (range, 45-75 year). The mean resected specimen length was $4.6 \mathrm{~cm}$ (range, 1.5-9.5 cm). The en block resection rate for the ESD was 100\%. Four patients had positive vertical resection margins, resulting in an en blockplus R0 resection rate of 82.6\% (19/23). Four other patients had LV invasion in the resected specimens. These eight patients (34.8\%) were regarded as incomplete treatment by the ESD and seven of them received adjuvant therapy. None had tumor recurrence during the mean follow-up of 30.1 months. Two of the remaining 15 patients with R0 resection without LV invasion eceived adjuvant therapy based on the protocol. The other 13 patients received close observations only, including 11 patients with SM2 cancers. None had tumor recurrence during the mean follow-up of 23.6 months.

Conclusions: ESD with/without adjuvant therapy based on the final pathological results may be an alternative treatment for patients with $\mathrm{pT} 1 \mathrm{~b}$ esophageal cancers.

\section{Introduction}

For superficial esophageal neoplasms (SENs), endoscopic submucosal dissection (ESD) is superior to conventional endoscopic mucosal resection in terms of curative treatment [1-3]. Currently, ESD is indicated for SENs confined to the mucosal layers (pT1a) [4]. Due to a substantial risk of lymph node metastasis (LNM), radical esophagectomy with lymphadenectomy is still regarded as the standard treatment for SENs invading to the submucosa (pT1b) [5]. However, to assess the depth of tumor invasion accurately before the ESD is difficult. Endoscopic ultrasound (EUS) is probably the most frequently used tool but it may not be sufficient to distinguish mucosal from submucosal cancers [6,7]. Therefore, it is inevitable that some patients with cT1aN0M0 SENs undergoing ESD would have pathologically upgraded $\mathrm{pT} 1 \mathrm{~b}$ cancers after the ESD. On the other hand, patients with cT1bN0M0 SENs may receive ESD rather than surgery because of high surgical mortality and morbidity rates, and poor quality of life after the surgery [8,9]. As a result, some of these patients may have pT1b cancers after the ESD. How to manage these patients with pT1b SENs after the ESD appropriately has not been well elucidated [10-13]. Therefore, we conducted this study to report the outcomes of the patients with pT1b esophageal cancers, with or without adjuvant therapy after the ESD.

\section{Patients and Methods}

\section{Publication History:}

Received: March 26, 2018

Accepted: June 23, 2018

Published: June 25, 2018

Keywords:

Adjuvant therapy, Endoscopic submucosal dissection, Lymph node metastasis, Lymphovascular invasion, $\mathrm{pT} 1 \mathrm{~b}$

\section{Study population}

A cohort of patients with superficial esophageal squamous cell carcinomas undergoing ESD in Chang Gung Memorial HospitalLinkou Medical Center was retrospectively identified from a computer database between May 2013 and November 2017. A total of 23 patients with 25 SENs who were diagnosed as pT1b cancers after the ESD were enrolled in this study. In our institution, the major indication for ESD was cT1aN0M0 SENs. However, those patients with cT1bN0M0 SENs who refused esophagectomy or concurrent chemoradiotherapy (CRT) also underwent ESD as a primary treatment. Therefore, the clinical stage was cTlaN0M0 in 17 patients (74\%), cT1bN0M0 in 4 patients (17.4\%), and cT1N0M0 in 2 patients $(8.6 \%)$.

"Corresponding Author: Dr. Yung-Kuan Tsou, Department of Gastroenterology and Hepatology, Chang Gung Memorial Hospital, 5, Fu-Shin Street, Kweishan, Taoyuan 333, Taiwan, Tel: 886-3-3281200, ext. 8108, Fax: 886-3-3272236; E-mail: flyidea3454@gmail.com

Citation: Tsou YK, Lee CH, Lee MS, Chuang WY, Lin CH, et al. (2018) Endoscopic Submucosal Dissection with/without Adjuvant Therapy for the Treatment of cT1N0M0 Esophageal Neoplasms Turned Out to be pT1b Cancers after the Endoscopic Resection. Int J Gastroenterol Disord Ther 5: 141. doi: https://doi.org/10.15344/2393-8498/2018/141

Copyright: ( 2018 Tsou et al. This is an open-access article distributed under the terms of the Creative Commons Attribution License, which permits unrestricted use, distribution, and reproduction in any medium, provided the original author and source are credited. 
Citation: Tsou YK, Lee CH, Lee MS, Chuang WY, Lin CH, et al. (2018) Endoscopic Submucosal Dissection with/without Adjuvant Therapy for the Treatment of cT1N0M0 Esophageal Neoplasms Turned Out to be pT1b Cancers after the Endoscopic Resection. Int J Gastroenterol Disord Ther 5: 141. doi: https://doi. org/10.15344/2393-8498/2018/141

Page 2 of 4

\section{Clinical staging}

Preoperative images for clinical staging included EUS, chest computed tomography (CT) scans, and integrated fluorodeoxyglucose positron emission tomography/CT (PET/CT). The invasion depth of the SEN was determined based on the results of EUS: cT1a was the SEN involving the mucosa but not the submucosa; cT1b was the SEN involving the submucosa but not the muscularis propria. Positive nodal staging by EUS was defined as lymph nodes more than $5-\mathrm{mm}$ in the shortest dimension that was spherical and had distinct borders, or by CT was those more than $10 \mathrm{~mm}$ in the shortest dimension [14]. The results of the three imaging studies were compared, and any discrepancies were resolved by consensus at a meeting following a further imaging review.

\section{ESD procedures}

All ESD procedures were performed by a single endoscopist (Tsou YK). The details of the ESD procedures were similar to those described in our previous reports $[15,16]$. The submucosal injection fluid was a glycerol solution plus indigo carmine with or without epinephrine $(0.0004 \%)$. ESD was performed mainly using an insulated tip 2 knife (KD-611L, Olympus). En bloc resection was defined as completed target resection in one piece.

\section{Pathological staging}

All specimens were pinned on a cork and fixed with $10 \%$ formalin. The specimens were embedded in paraffin, cut into parallel 2-mm serial sections, and stained with hematoxylin and eosin. All specimens were examined microscopically for histological type, depth of invasion, lymphovascular (LV) invasion, and resection margins by an experienced pathologist (Chuang WY). The depth of invasion in submucosa was divided into SM1 when the tumor infiltrating the submucosa up to $200 \mu \mathrm{m}$; and SM2, when the tumor invading more than $200 \mu \mathrm{m}$ [4]. Completeness of resection was expressed as R0, when all resection margins were free of tumor cells.

\section{Adjuvant therapy and Follow-up}

Adjuvant therapy included esophagectomy with lymphadenectomy or concurrent CRT. It was our protocol to give the patients adjuvant therapy after the ESD for the following criteria: (1) positive vertical resection margins; (2) presence of $\mathrm{LV}$ invasion in the resected specimens; or (3) any cancer with SM2 invasion. To receive surgery or CRT was the patient's discretion. If the patient refused adjuvant therapy, close observations were offered.

The adjuvant CRT consisted of two cycles of cisplatin $60 \mathrm{mg} / \mathrm{m} 2$ on day 1 , and continuous infusion of 5 -fluorouracil $1,000 \mathrm{mg} / \mathrm{m} 2 /$ day on day 1 to 3 , repeated every 4 weeks with concurrent radiotherapy of 45 Gy in more than 20 fractions.

During the follow-up period, esophagogastroduodenoscopy with narrow band images, with or without Lugol's staining was carried out every 3-6 months and chest CT scans every 6-12 months for all patients. The metachronous neoplasm was defined as a neoplasm that was detected at the esophagus other than the resection site (the scar area). Local recurrence was defined as a neoplasm that was detected at the site of resection and con-firmed histologically. Recurrent tumor included local recurrence, LNM, or distant metastasis. The follow-up data were updated in December 2017.

\section{Results}

The patients who had more than one SEN were classified according to the SEN with the greatest invasion depth. The clinical features of the patients are listed in Table 1. The mean patient age was 56.3 years; 22 patients $(95.7 \%)$ were man. Pre-existing major comorbidities included concurrent cancers $(n=10,43.5 \%)$, previous cancers $(n=9,39.1 \%)$, and liver cirrhosis $(n=8,34.8 \%)$. The concurrent or previous cancers were mainly head and neck cancers. All patients had an Eastern Cooperative Oncology Group performance status score of 0 or 1 [17].

\begin{tabular}{|c|c|}
\hline & Total \\
\hline & $(\mathrm{n}=23)$ \\
\hline Mean age, yr (range) & $56.3(45-75)$ \\
\hline Gender, man & $22(95.7 \%)$ \\
\hline \multicolumn{2}{|c|}{ Pre-existing comorbidities } \\
\hline Concurrent cancers & $10(43.5 \%)$ \\
\hline Previous cancers & $9(39.1 \%)$ \\
\hline Liver cirrhosis & $8(34.8 \%)$ \\
\hline \multicolumn{2}{|l|}{ Clinical stage } \\
\hline cT1aN0M0 & $17(74 \%)$ \\
\hline cT1bN0M0 & $4(17.4 \%)$ \\
\hline cT1N0M0 & $2(8.6 \%)$ \\
\hline
\end{tabular}

The results of the ESD are listed in Table 2. None of the patients experienced serious complications such as major bleeding or perforation which were related to the ESD. The mean resected specimen length was $4.6 \mathrm{~cm}$. Theen block resection rate was $100 \%$. Theen block plus R0 resection rate was $82.6 \%(19 / 23)$ due to four patients with positive vertical resection margins (all were SM2 cancers). As to the invasion depth, four patients (17.4\%) had SM1 cancers and 19 patients $(82.6 \%)$ had SM2 cancers. The mean depth of tumor invasion was $0.79 \mathrm{~mm}$ (range, $0.1-1.6 \mathrm{~mm}$ ). With regard to the histological grading, none had well-differentiated cancers, nine patients (39.1\%) had moderately-differentiated cancers, and 14 patients (60.9\%) had poorly-differentiated cancers. Four patients $(17.4 \%$, all with R0 resection) had LV invasion in the resected specimens.

The outcomes of the patients after the ESD were demonstrated in Figure 1. According to the protocol, seven of the eight patients with positive resection margin or LV invasion in the specimens received adjuvant therapy (four for surgery and three for CRT). The other one patient (positive vertical resection margin) refused adjuvant therapy. He was followed-up closely and died of pneumonia $35^{\text {th }}$ month after the ESD. There was no recurrent tumor during the mean follow-up period of 30.1 months in this group of patients. Two of the remaining 15 patients with $\mathrm{R} 0$ resection without $\mathrm{LV}$ invasion received adjuvant therapies (one for surgery and the other for CRT) according to the protocol. The other 13 patients $(56.5 \%, 11$ with SM2 cancers) received close observations only. Two of the patients died of the concurrent head and neck cancers $5^{\text {th }}$ month after ESD, respectively. One other patient died of pneumonia $21^{\text {st }}$ month after the ESD. There was no recurrent tumor during the mean follow-up period of 23.6 months in this group.

Of the five patients received adjuvant surgery, the average number of dissected lymph nodes was 37.8 (range, 30-47). Only one patient (20\%) had tumor cells infiltration in one of the dissected lymph nodes. 
Citation: Tsou YK, Lee CH, Lee MS, Chuang WY, Lin CH, et al. (2018) Endoscopic Submucosal Dissection with/without Adjuvant Therapy for the Treatment of cT1N0M0 Esophageal Neoplasms Turned Out to be pT1b Cancers after the Endoscopic Resection. Int J Gastroenterol Disord Ther 5: 141. doi: https://doi. org/10.15344/2393-8498/2018/141

Page 3 of 4

\begin{tabular}{|c|c|}
\hline & Total \\
\hline & $(\mathrm{n}=23)$ \\
\hline Mean resected specimen length, $\mathrm{cm}$ & $4.6(1.5-9.5)$ \\
\hline En block resection & $23(100 \%)$ \\
\hline En block plus R0 resection & $19(82.6 \%)$ \\
\hline Positive horizontal margins & 0 \\
\hline Positive vertical margins & $4(17.4 \%)$ \\
\hline \multicolumn{2}{|l|}{ The depth of tumor invasion } \\
\hline SM1 & $4(17.4 \%)$ \\
\hline SM2 & $19(82.6 \%)$ \\
\hline Mean depth of SM invasion, mm (range) & $0.79(0.1-1.6)$ \\
\hline \multicolumn{2}{|l|}{ Histological grading } \\
\hline G1 & 0 \\
\hline G2 & $9(39.1 \%)$ \\
\hline G3 & $14(60.9 \%)$ \\
\hline Presence of lymphovascular invasion & $4(17.4 \%)$ \\
\hline \multicolumn{2}{|c|}{$\begin{array}{l}\text { Table 2: The results of the endoscopic submucosal dissection. } \\
\text { Abbreviations: SM1: tumor invading the SM up to } 200 \mu \mathrm{m} \text {; SM2: } \\
\text { tumor invading the SM more than } 200 \mu \mathrm{m} \text {; }\end{array}$} \\
\hline
\end{tabular}

\section{Discussions}

Adjuvant therapy is required for some of the patients with pT1b esophageal cancers after the ESD because of the risks of LNM which is not identified by pre-ESD imaging studies. The risk of LNM for $\mathrm{pT} 1 \mathrm{~b}$ was reported to be $25.9-53.8 \%$ [18-20]. However, these data were from retrospective surgical series. Applying these surgical data to the patients undergoing ESD with the results of pT1b may overestimate the risk of LNM for the following reasons. First, the surgical series included the patients with any nodal status but for the ESD, only patients with negative nodal status were enrolled. Shin et al. reported that the risk of unexpected LNM was only $9.7 \%(7 / 72)$ for the patients with cT1aN0M0 SENs undergoing surgery [21]. Second, the surgical series included SENs with deep submucosal invasion. In this study, the mean depth of tumor invasion in the submucosal layer was 0.79 $\mathrm{mm}$, implying that the patients undergoing ESD with the results of pT1b may have shallower submucosa invasion. Araki et al. reported that the incidence of LNM increased from $13.0 \%$ to $58.3 \%$ as the depth of tumor invasion in SM layer ranged from $<1 \mathrm{~mm}$ to $>2 \mathrm{~mm}$, respectively [20]. Third, surgically resected specimens were usually cut into 5 -mm slices, which was much thicker than the 2-mm slices of ESD specimens $[19,20]$. Thick sliced specimens may result in under stage of the SEN, leading to overestimation of the rate of LNM for a particular depth of invasion.

A literature review revealed that LV invasion and invasion depth by the tumors were the two common independent risk factors for LNM of SENs $[18,22,23]$. Miura et al. reported that LV invasion was the most important factor for prediction of LNM [24]. In this study, the mean depth of tumor invasion in the submucosal layer in the patients with positive vertical resection margin was $1.2 \mathrm{~mm}$ (range, 0.8$1.5 \mathrm{~mm})$, deeper than those with free margins $(0.69 \mathrm{~mm}, \mathrm{p}=0.025)$. In other words, positive vertical resection margins indicated not only the possibility of incomplete treatment but also the tumor infiltration to the deeper SM. Therefore, the patients with either one of the two risk factors after the ESD should receive adjuvant therapy.

Either surgery or CRT could be the adjuvant therapy $[10,13,25]$. Adjuvant surgery resulted in good oncological outcomes but it did cause morbidity and even mortality in the patients. In an abstract form, Muto et al. reported that endoscopic resection combined with prophylactic CRT resulted in a 3-year overall survival of $90.7 \%$ for the 87 patients with pT1b with negative resection margin and pT1a with vascular invasion [25]. Ikeda et al. reported that the patients with adjuvant therapy (surgery or chemoradiotherapy) had a better 3-year relapse-free survival rate than the patients without ( $88 \%$ vs. $64 \%)$ [13]. However, in this study, whether the patients underwent adjuvant therapy or not were based on the doctor's discretion or the patient's

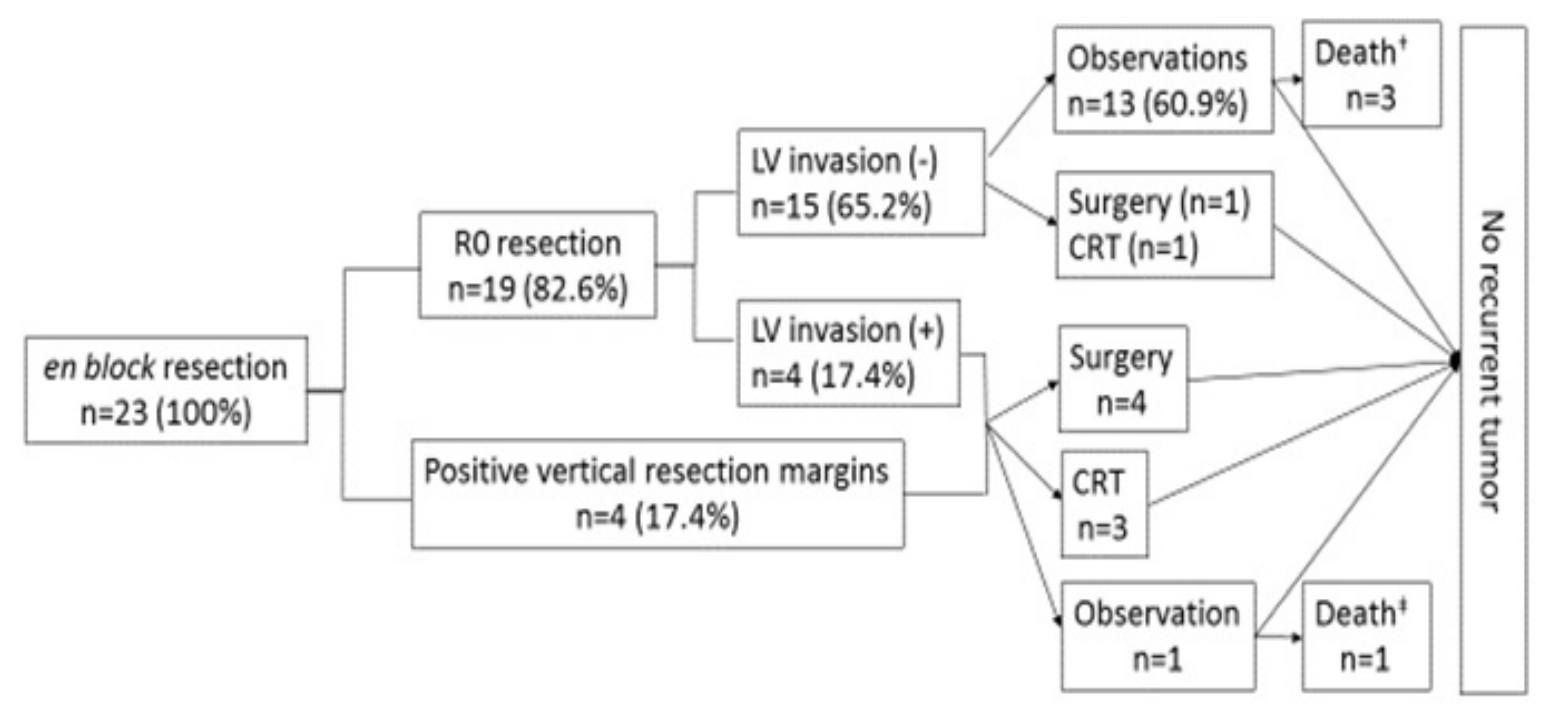

Figure 1: Outcomes of the patients with or without adjuvant therapy.

two patients died of Head and Neck cancers at $5^{\text {th }}$ month, one died of pneumonia at $12^{\text {th }}$ months

*died of pneumonia at $35^{\text {th }}$ months

Abbreviations: LV: lymphovascular; CRT: chemoradiotherapy 
Citation: Tsou YK, Lee CH, Lee MS, Chuang WY, Lin CH, et al. (2018) Endoscopic Submucosal Dissection with/without Adjuvant Therapy for the Treatment of cT1N0M0 Esophageal Neoplasms Turned Out to be pT1b Cancers after the Endoscopic Resection. Int J Gastroenterol Disord Ther 5: 141. doi: https://doi. org/10.15344/2393-8498/2018/141

Page 4 of 4

preference but not the risk of LNM after the ESD. The patients without risk of metastasis might have received unnecessary adjuvant therapy and the patients with risk of metastasis might be undertreated.

The major limitation of the present study is its retrospective design with a relatively small case number from a single center. Prospective, large-scale studies are required to confirm our observations.

In conclusion, ESD with/without adjuvant therapy based on the pathological findings after the ESD may be an alternative treatment for patients with pT1b SENs but needs further study to confirm.

\section{Competing Interests}

The authors have declared that no competing interest exists.

\section{References}

1. Takahashi H, Arimura Y, Masao H, Okahara S, Tanuma T, et al. (2010) Endoscopic submucosal dissection is superior to conventional endoscopic resection as a curative treatment for early squamous cell carcinoma of the esophagus (with video). Gastrointestinal Endoscopy 72: 255-264.

2. Oyama T, Tomori A, Hotta K, Morita S, Kominato K, et al. (2005) Endoscopic submucosal dissection of early esophageal cancer. Clinical Gastroenterology \& Hepatology 3: 67-70.

3. Fujishiro M, Yahagi N, Kakushima N, Kodashima S, Muraki $\mathrm{Y}$, et al (2006) Endoscopic submucosal dissection of esophageal squamous cell neoplasms. Clin Gastroenterol Hepatol 4: 688-694.

4. Kuwano H, Nishimura $\mathrm{Y}$, Oyama T, Kato H, Kitagawa $\mathrm{Y}$, et al. (2015) Guidelines for Diagnosis and Treatment of Carcinoma of the Esophagus April 2012 edited by the Japan Esophageal Society. Esophagus 12: 1-30.

5. Wang GQ, Jiao GG, Chang FB, Fang WH, Song JX, et al. (2004) Long-term results of operation for 420 patients with early squamous cell esophageal carcinoma discovered by screening. The Annals of thoracic surgery 77 1740-1744.

6. Bergeron EJ, Lin J, Chang AC, Orringer MB, Reddy RM, et al. (2014) Endoscopic ultrasound is inadequate to determine which T1/T2 esophageal tumors are candidates for endoluminal therapies. Journal of Thoracic \& Cardiovascular Surgery 147: 765-771.

7. Polkowski M (2009) Endosonographic staging of upper intestinal malignancy. Best Practice \& Research Clinical Gastroenterology 23: 649661.

8. Carr SR, Jobe BA (2010) Esophageal preservation in the setting of high-grade dysplasia and superficial cancer. Seminars in thoracic and cardiovascular surgery 22: 155-164

9. Derogar M, Orsini N, Sadr-Azodi O, Lagergren P (2012) Influence of major postoperative complications on health-related quality of life among longterm survivors of esophageal cancer surgery. Journal of clinical oncology : official journal of the American Society of Clinical Oncology 30: 1615-1619.

10. Motoyama S, Jin M, Matsuhashi T, Nanjo H, Ishiyama K, et al. (2013) Outcomes of patients receiving additional esophagectomy after endoscopic resection for clinically mucosal, but pathologically submucosal, squamous cell carcinoma of the esophagus. Surgery Today 43: 638-642.

11. Mochizuki Y, Saito Y, Tsujikawa T, Fujiyama Y, Andoh A, et al. (2011) Combination of endoscopic submucosal dissection and chemoradiation therapy for superficial esophageal squamous cell carcinoma with submucosal invasion. Experimental and Therapeutic Medicine 2: 10651068.

12. Kawaguchi G, Sasamoto $R$, Abe E, Ohta A, Sato H, et al. (2015) The effectiveness of endoscopic submucosal dissection followed by chemoradiotherapy for superficial esophageal cancer. Radiation Oncology 10: 31

13. Ikeda A, Hoshi N, Yoshizaki T, Fujishima Y, Ishida T, et al. (2015) Endoscopic Submucosal Dissection (ESD) with Additional Therapy for Superficia Esophageal Cancer with Submucosal Invasion. Internal Medicine 54: 28032813.
14. Shimizu Y, Tsukagoshi H, Fujita M, Hosokawa M, Kato M, et al. (2002) Long-term outcome after endoscopic mucosal resection in patients with esophageal squamous cell carcinoma invading the muscularis mucosae or deeper. Gastrointest Endosc 56: 387-390.

15. Tsou YK, Chuang WY, Liu CY, Ohata K, Lin CH, et al. (2016) Learning curve for endoscopic submucosal dissection of esophageal neoplasms. Diseases of the esophagus : official journal of the International Society for Diseases of the Esophagus 29: 544-550.

16. Tsou YK, Liu CY, Fu KI, Lin CH, Lee MS et al (2016) Endoscopic Submucosal Dissection of Superficial Esophageal Neoplasms Is Feasible and Not Riskier for Patients with Liver Cirrhosis. Digestive diseases and sciences 61: 3565-3571.

17. Oken MM, Creech RH, Tormey DC, Horton J, Davis TE, et al. (1982) Toxicity and response criteria of the Eastern Cooperative Oncology Group. American journal of clinical oncology 5: 649-655.

18. Li B, Chen H, Xiang J, Zhang Y, Kong Y, et al. (2013) Prevalence of lymph node metastases in superficial esophageal squamous cell carcinoma. The Journal of Thoracic and Cardiovascular Surgery 146: 1198-1203.

19. Eguchi T, Nakanishi Y, Shimoda T, Iwasaki M, Igaki H, et al. (2006) Histopathological criteria for additional treatment after endoscopic mucosal resection for esophageal cancer: analysis of 464 surgically resected cases. Mod Pathol 19: 475-480.

20. Araki K, Ohno S, Egashira A, Saeki H, Kawaguchi H, et al. (2002) Pathologic features of superficial esophageal squamous cell carcinoma with lymph node and distal metastasis. Cancer 94: 570-575.

21. Shin S, Kim HK, Choi YS, Kim K, Shim YM, et al. (2014) Clinical stage T1T2NOMO oesophageal cancer: accuracy of clinical staging and predictive factors for lymph node metastasis. Eur J Cardiothorac Surg 46: 274-279.

22. Jia R, Luan Q, Wang J, Hou D, Zhao S, et al. (2016) Analysis of Predictors for Lymph Node Metastasis in Patients with Superficial Esophageal Carcinoma. Gastroenterology Research and Practice 2016: 6.

23. Kim DU, Lee JH, Min BH, Shim SG, Chang DK, et al. (2008) Risk factors of lymph node metastasis in T1 esophageal squamous cell carcinoma. $J$ Gastroenterol Hepatol 23: 619-625.

24. Miura A, Momma K, Yoshida M (2009) Endoscopic resection for T1a-MM and T1b-SM1 squamous cell carcinoma of the esophagus. Clinical journal of gastroenterology 2: 252-256.

25. Muto M, Minashi K, Nihei K, Mizusawa J, Yano T, et al. (2016) Efficacy of combined endoscopic resection and chemoradiotherapy for clinical stage $\mid$ esophageal squamous cell carcinoma (ESCC): A single-arm confirmatory study (JCOG0508). Journal of Clinical Oncology 34: 4013-4013. 